\title{
Selective and sensitive smart grid with software defined control towards sustainable power quality
}

\author{
Deepak Guru $\mathrm{G}^{\mathrm{a}}$, Prema $\mathrm{M}^{\mathrm{b}}$, Nivetha $\mathrm{R}^{\mathrm{b}}$, Chandrasekaran $\mathrm{S}^{\mathrm{a}, \mathrm{b}} *$ \\ ${ }^{a}$ Department of Mechatronics Engineering,Sri Krishna College of Engineering and Technology,Kuniamuthur,Coimbatore- \\ 641008, India. \\ ${ }^{b}$ Department of Electrical and Electronics Engineering,Sri Krishna College of Engineering and \\ Technology,Kuniamuthur,Coimbatore-641008,India.
}

\begin{abstract}
The objective of the research work is to propose a selective and sensitive smart grid model with software defined control techniques to achieve sustainable power quality. The existing smart grid techniques do not provide a high level of accuracy in optimizing the power switched to the consumers when the number of appliances are massive.The change-over time to different power lines across multiple devices is to be minimal and safe. This basic infrastructure has to be secured against un-exploitable cyber-attacks on necessary services that may create severe damage on human life. A framework with smart switching based on individual demand sensing and distributing the required supply to the needed energy customer is proposed with the help of server based software applications
\end{abstract}

Keywords: Smart switching, distributed control, smart meters, protection, intelligence sensing

\section{Introduction}

There are many technical and economic issues and challenges to be faced in rendering the essential power supply service connections even to remote areas in developing countries like India. The population growth and density creates a major social and governance problem in designing and erecting a smart grid for a mammoth democratic nation with multiple races and geographic obstacles like hilltop towns and valley villages. The important feature of such electric power grid is to be a strict regulation and enforcement authorization to prevent any major energy accidents and minor power thefts. The average power theft in various Indian states are mentioned in Table 2 due to the nonadvancement of electric power grids. The characteristic feature of a smart grid is two-way flow of electricity and information which incorporates the benefits of delivering real time information and enable balance of supply and demand at device level [1].Today's grid varies by a lot of aspects than the smart grid. In today's grid there is only limited consumer participation, dominated by central generation, not well integrated and has a slow response to power quality issues. Whereas smart grid on the other hand has more involved consumers, distributed energy resources, is well integrated and responds rapidly to power quality issues [2].Generation of renewable energy is very important for managing the energy crisis and for the sustainable development of the society. But the generation is uncertain and interconnecting these generated renewable resources with the grid becomes so difficult.

Considering all these factors, in the future, a smart grid must be laid in order to provide a dynamic platform for safe interconnection of a renewable energy system to the grid [3].Maximum utilization of renewable energy is possible by customer owned installation called a 'micro grid' which includes both generation and consumption of power from the grid. The difference in average power consumption in

\footnotetext{
* Manuscript received Feb. 2, 2018; revised June 5, 2018.

Corresponding author. Tel.: 9488790722;E-mail address: deepakguru1998@gmail.com.

doi: $10.12720 /$ sgce.7.3.180-187
} 
various states of India during normal and peak hours is mentioned in Table 2. In order to meet the demand during peak hours, an electric power grid with effective integration of renewable energy resources must be employed. It provides an enhanced method of demand management, better efficiency and reduced transmission and distribution costs. Advanced metering infrastructure (AMI) also called smart meters when incorporated with above technologies will enhance the quality of the smart grid [4].It makes it more sustainable and reliable. A solution proposed to improve the quality of a smart grid is a tie set graph theory, which involves dividing the power system into loops for simpler synchronization and reduced loss of electricity. Another aspect which includes smart grid and renewable energy is the Optimal Real time Distribution of renewable Energy Resources (ORDER) problem, which is basically based on a concept where power is distributed between points having excessive power and points having little power [5]. The important challenges are the usage of diversified multi branded uncertified low cost electrical appliances and pinning up of illegal random supply connections for unscheduled local festivals and gatherings over a lengthy period of time. The political and social issue can be solved by a stack of software defined power control stack through which any sort of power consumption can be monitored and regulated using this distributed control over the national power grid. At the same time, the present smart grid techniques face many communication and computing challenges in data handling and information securing systems. The critical data will flood into servers from different types of end consumers of energy towards their timely billing. All these data has to be maintained with high confidentiality with a dynamic data flow encryption techniques. The back end servers and the grids are the only protected entities by the firewalls, the meters at the consumer end should also be protected by either encryption or obfuscation techniques. The cyber-attacks may be initiated at any point to the extent till the transformers perimeter and small electrical appliances.

Table 1.Power consumption in various regions [6].

\begin{tabular}{lcl}
\hline Indian power region & Requirement at normal hours & Requirement at peak hours \\
\hline Northern & 27300 & 44200 \\
\hline Western & 29400 & 45300 \\
\hline Southern & 23800 & 37400 \\
\hline Eastern & 9900 & 17200 \\
\hline North eastern & 12000 & 2400 \\
\hline Total & 91600 & 146500 \\
\hline
\end{tabular}

Table 2. Average power theft[7].

\begin{tabular}{ll}
\hline Indian states & Average power theftin percentage \\
\hline Jammu and Kashmir & 63 \\
\hline Bihar & 39 \\
\hline Jharkhand & 38 \\
\hline Uttar Pradesh & 36 \\
\hline Rajasthan & 32 \\
\hline All India & 22.1 \\
\hline
\end{tabular}

The requirement of power at normal hours and peak hours in various Indian regions is tabulated from the details collected from Statistics as per Ministry of Power, Government of India. 


\section{Architecture of Sensitive Smart Gird}

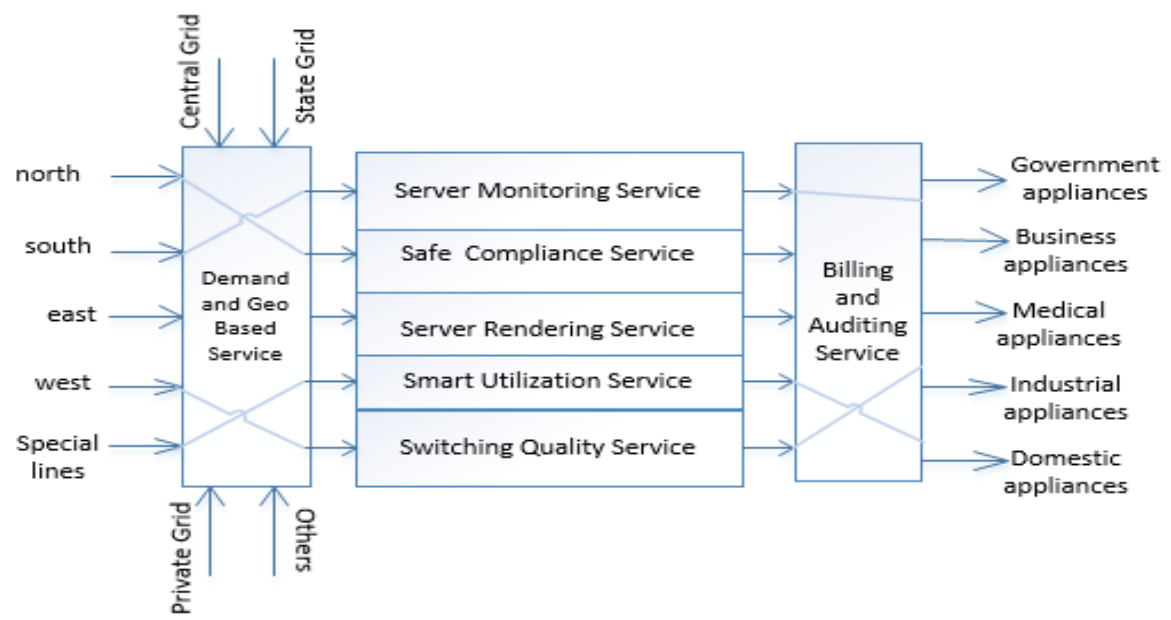

Fig. 1. Architecture of Sensitive Smart Grid

The generation and transmission of electricity in smart grid is mainly carried out in series of service layers as shown in Figure 1. First, the electricity is received from various sources like central, state and private grids and from renewable resources. The demand and geo based sensing process is carried out and thus, data is collected on how much electricity is needed to each place. Secondly, there are many interim processes that are carried out before delivering the electricity to the required places. They are

- Server Monitoring Service: The server has to be monitored 24/7 to ensure balanced demand and supply. This monitoring must also indicate any faults occurring in the grid and automatic rectification must be carried out within microseconds.

- Safe Compliance Service: The quality of electricity that is supplied must be within the standard limits as prescribed by the legislation and compliance authorities. This service ensures that safe compliance is provided.

- Server Rendering Service: The client requests the server for the amount of electricity it needs. The server must be able to process the request and respond to it very quickly. In order to have a better customer care this server rendering service should be present in a smart grid.

- Smart Utilization Service: To enable the load balancing between the regions and to satisfy the peak demand in the early evening hours, the smart utilization service must be used in order to reap the full potential of smart grids. This service can be enabled by constructing smart grids at regional levels thus reducing the transportation losses.

- Switching Quality Service:Depending upon the requirement, the smart grid is able to send the electricity to a particular place rather than sending it to the other places. All the electricity must be able to be diverted within microseconds and for this the switching quality must be of high standards.After all these processes are carried out, the electricity is sent to the required place. The tariff governance and auditing work has to be carried out for the amount of electricity supplied to each place based on the type of application (Domestic, Commercial, Industrial applications).All these parameters as a whole comprises a better and enhanced smart grid.

\section{Tree of Smart Grid Quality Factors}

The smartness of the grid is classified based on various Quality Factors. The main two classification factors are sensitivity and selectivity. As shown in figure 2 , sensitivity is further classified based on the 
demand factors, generation factors and transmission factors. The demand factors depend upon the amount of electric power required for a certain application for a certain period of time. The demand factor is also based on the erection and operational cost of the grid. The generation factors are based upon the type of resources used for generation of electricity and the safety requirements during the generation. The transmission factors have important feature of on-demand auto switching and ondemand manual switching based upon the user requirement. During the peak hours based upon the demand and requirement and based on software defined control the switching process is carried out. The transmission factors further concentrate on safety of the Smart Grid against cyber-attack and system vulnerability.

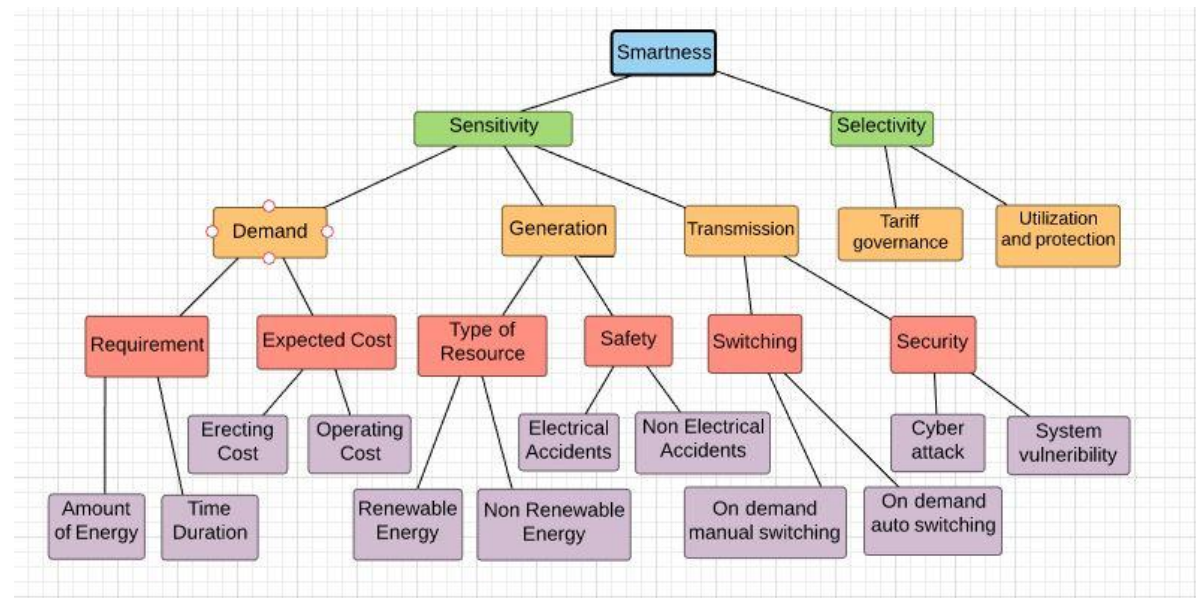

Fig. 2. Tree of Smart Grid Quality Factors

\section{Methodology}

Computational Model of the existing ORDER (Optimal Real Time Distributed Energy Resources) can be upgraded with the introduction of various regulation features. These regulatory acts will corresponding to the essential compliance and enforcement strategies in the generation, utilization and consumption stages of distributed energy resources. Thereby an improvised and monitored ordering can be achieved leading to REORDER technique (Regulation Enforcement ORDER).The compliance and enforcement strategies can be categorized into the following categories:Safety and Security Regulations comprises of various safety regulationslike insulation of wires and security regulations against cyber-security attacks. Consumption and Maintenance Regulations must regulate the amount of energy consumed and the power line must be maintained in accordance with the regulation. Tariff and Metering Regulations are used for regulating the cost for the consumed energy for different end consumers based on their applications. Monitoring and Emergency Regulations consists of measures to be taken during emergency situations and monitoring of the grids.

As per the earlier order problem, for a given time sequence $T=\{t\}$, each $v \in V$, and each $e(v, u) \in E$, decides the amount of power by CGF $\operatorname{Dv}(t)$ and the edge flow $f(v, u, t)$ that satisfies the following conditions with certain threshold $\mathrm{P}_{\min }$.

Maximize $\max _{v \in V}\left\{P_{v}(t)\right\}$

Minimize $\sum_{t \in T} \sum_{v \in V} D_{v}(t)$

$P_{v}(t) \geq P_{\min }$

In the proposed RE-ORDER technique, the regulation compliances and enforcement directives are to 
be applied across the nodes through the edges. The safety and security compliances and smart metering standards are cross checked as conditions across loops. The conditionals are so emphasized since various regulatory measures and standards are needed for different geo power stations. The distributed energy sources are to be monitored not only according to transmission standards but also for the storage regulations.

Maximize $\max _{v \in V}\left\{P_{v}(t)\right\} \mid R_{v}(t)(1 \mathrm{~b})$

Minimize $\sum_{t \in T} \sum_{v \in V} D_{v}(t) \mid E_{v}(t)(2 \mathrm{~b})$

There are two different categories of energy sources. One category comprises of renewable energies and other category of non-renewable energies. The renewable energy sources are tidal, wind, bio and solar energies. The non-renewable energy sources are thermal, nuclear and hydroelectric energies.

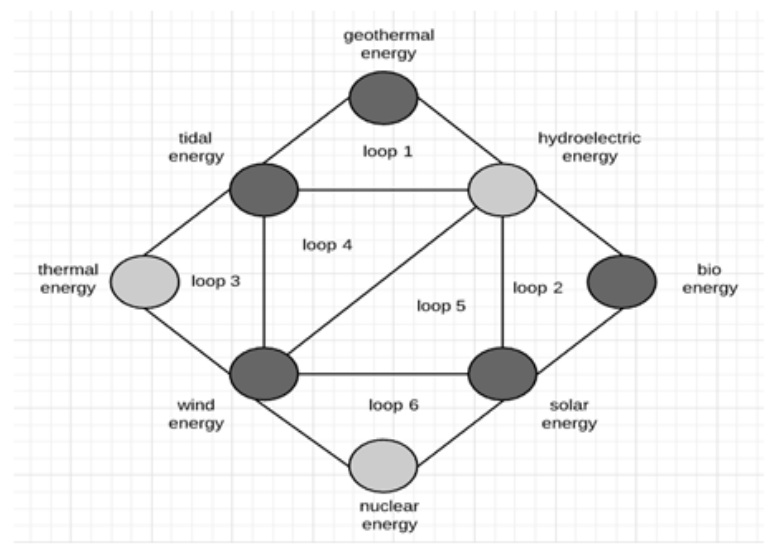

Fig. 3. Source of energy set

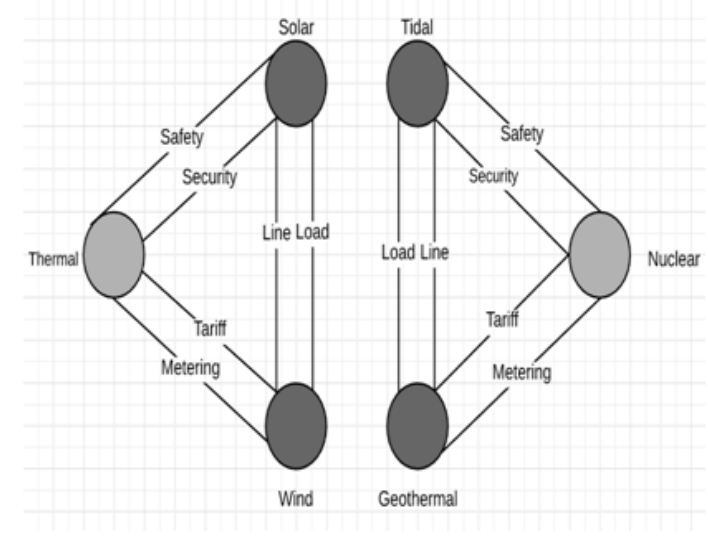

Fig. 4. Loop 1 and Loop 2

$\mathrm{NR}=\{$ thermal, nuclear, hydroelectric $\} \Rightarrow\{$ th, nu,hy $\}$

$\mathrm{R}=\{$ tidal, bio, wind, solar, geothermal $\}=>\{\mathrm{ti}, \mathrm{bi}, \mathrm{wi}, \mathrm{so}, \mathrm{ge}\}$

NR represents Non-renewable energy resources

$\mathrm{R}$ represents Renewable energy resources.

All the sources of the energy can be represented in the form of a graph, $G=(N, E)$.In the sources of energy set as given in figure 3, the graph is represented as a set of nodes and edges. The nodes represent the distributed energy sources and the edges represent the regulation and enforcement measures between the vertices. There are sets of nodes and edges in the graph.We can divide thisgraph into set of 3 nodes called loops. Each loop must contain one non-renewable energy resource and two renewable energy resources. For example loop 1 in figure 4 contains thermal, solar and wind energy out of which thermal is non-renewable and other two are renewable energy resources.

Loop1 $=>\{$ th ,so,wi $\}$, Loop2=>\{nu,ti,ge $\}$

This type of loops are formed in-order to have coordination and cooperation from different sources of energy to have a balanced grid. This source of energy set can be extended to any number of loops comprising of any number of energy resources.

\subsection{Inter-operability and integrity}

Considering one of the loops from the source of energy set graph, the nodes and the vertices are addressed from the Figure 4 below. The regulatory and enforcement relations across the energy nodes are also shown. For example, in loop 1, the non-renewable energy source is being the thermal station with a 
maximum 1200 MW and the renewable energy sources like Wind mill station of about 100MW and Solar power station is capable of generating $250 \mathrm{MW}$. But in this loop 1, the various regulation and enforcement responsibilities will be looked after by every other node in the same loop.

Table 3. Energy sources in Loop 1 and regulations

\begin{tabular}{|c|c|c|c|c|c|c|}
\hline $\begin{array}{l}\text { Regulation } \\
\text { Sources }\end{array}$ & Safety & Security & Line & Load & Metering & Tariff \\
\hline Thermal & $\checkmark$ & $\checkmark$ & & & & \\
\hline Wind & & & & & $\checkmark$ & $\checkmark$ \\
\hline Solar & & & $\checkmark$ & $\checkmark$ & & \\
\hline
\end{tabular}

Table 4.Energy sources in loop 2 and regulations

\begin{tabular}{|c|c|c|c|c|c|c|}
\hline Sources & Safety & Security & Line & Load & Metering & Tariff \\
\hline Tidal & & & $\checkmark$ & $\checkmark$ & & \\
\hline Geothermal & & & & & $\checkmark$ & $\checkmark$ \\
\hline Nuclear & $\checkmark$ & $\checkmark$ & & & & \\
\hline
\end{tabular}

Table 3 and Table 4 shows the different regulations being monitored by different sources of energies in each loop. For example as per the table 3a, thermal station monitors the safety and security regulation, windmill stations look after metering and tariff regulation, solar power plants regulate line and load regulations. Table $3 \mathrm{~b}$ shows that tidal stations look after line and load regulations, geothermal stations regulate metering and tariff, nuclear stations monitor safety and security regulations. These regulations are monitored by each stations based upon the software defined control from the controller through cloud based services.

$$
\begin{aligned}
& i_{o p}=\frac{\text { Energy } \text { Transmitted }+E_{\text {loss }}}{E_{\text {generated }}+E_{\text {stored }}} \\
& i_{\text {in }}=\frac{N_{R}+E_{\text {consumed }}}{N_{\text {sources }}+N_{\text {stored }}} \\
& \text { Selectivity }=\frac{N_{R} \times i_{o p}}{N_{R}+N_{N R}} \\
& \text { Sensitivity }=\frac{E_{R} \times i_{\text {in }}}{E_{R}+E_{N R}}
\end{aligned}
$$

Iop represents the interoperability for the exchange of deficient power between the renewable and nonrenewable energy resources. Interoperability is directly proportional to the summation of amount of energy transmitted and amount of loss of energy and is indirectly proportional to the summation of amount of energy generated and stored. iinrepresents the interchangeability of the renewable sources with the existing sources of Smart grid. Integrity is directly proportional the product of number of renewable resources and amount of energy consumed and is inversely proportional to the product of number of energy resources and amount of energy stored. The overall real time efficiency and utility is determined 
by the sensitivity and selectivity of interoperable and interchangeable energy resources which not only should be regulated but also the legal laws should be enforced. The selectivity is defined as the ability of the smart grid respond for a particular energy request from clients outside the grid and also the peers inside the grid. The selectivity is directly proportional to the product of number of renewable energy resources and interoperability and is inversely proportional to the summation of number of renewable and non-renewable energy resources. The sensitivity of the grid can be defined as the amount of energy that can be combined with the other sources of energy. It is directly proportional to the product of amount of renewable energy resources and interchangeability and is inversely proportional to the summation of amount of renewable and non-renewable sources of energy. Software Defined Control (SDC) techniques focuses on intelligent sensing and switching to the needed appliances in the specific areas of demand with maximum safety and security .The research work is mainly focused on aspects like demand sensing, generation sensitivity, transmission selectivity, utilization security, protection compliance and tariff governance. Generally the power requirement at any demanding point at any instant of time, is to be sensed with the type of supply needed to be selected at each place. Based upon the demand the supply is identified with all its generation parameters like area, type of energy, minimum and maximum permissible capacity of power. The supply selection and grid switching is carried out in a smart way with maximum safety and security by verifying power transmission compliances and appliance regulatory certifications. To ensure the end to end safety, the supply and its utilization must also be monitored periodically.

Table 5. Renewable energy sources with regulated generation and enforced transmission stages[8]

\begin{tabular}{lll}
\hline $\begin{array}{l}\text { Source wise all India } \\
\text { Generation from renewable }\end{array}$ & December 2016 & December 2015 \\
\hline Wind & 1779.89 & 1498.01 \\
\hline Solar & 1157.9 & 662.04 \\
\hline Biomass & 333.48 & 355.56 \\
\hline Bagasse & 1781.57 & 1912.04 \\
\hline Small Hydro & 339.19 & 528.56 \\
\hline Others & 50.51 & 19.6 \\
\hline Total & 5442.54 & 4975.81
\end{tabular}

From the Table 5 it is observed that the generation of power from renewable energy sources has been increased from 4975.81MW to 5442.54 MW in the year 2015-16. This increase in generation of electric power has been achieved by adapting regulation and enforcement laws, and the same is incorporated in this research paper.

Table 6. Non-Renewable energy sources with regulated generation and enforced transmissionstages[9]

\begin{tabular}{|c|c|c|c|c|c|}
\hline Year & Coal & Lignite & $\begin{array}{l}\text { Natural } \\
\text { gas }\end{array}$ & Electricity & Total \\
\hline 2006-07 & 6995 & 350 & 1448 & 1614 & 16571 \\
\hline 2007-08 & 7608 & 394 & 1533 & 1807 & 17878 \\
\hline 2008-09 & 8315 & 362 & 1533 & 1994 & 18936 \\
\hline $2009-10$ & 8856 & 391 & 2144 & 2206 & 21408 \\
\hline $2010-11$ & 8952 & 429 & 2357 & 2500 & 22458 \\
\hline 2011-12 & 9723 & 476 & 2299 & 2827 & 23872 \\
\hline 2012-13 & 10421 & 523 & 2038 & 2967 & 25128 \\
\hline 2013-14 & 10957 & 499 & 1836 & 3147 & 25756 \\
\hline 2014-15 & 12435 & 534 & 1859 & 3415 & 27589 \\
\hline 2015-16 & 12595 & 483 & 1843 & 3604 & 28276 \\
\hline $\begin{array}{c}\text { Growth rate of } \\
2015-16 \text { over } \\
2014-15(\%)\end{array}$ & 1.29 & -9.41 & -0.84 & 5.55 & 2.49 \\
\hline $\begin{array}{l}\text { CAGR 2006-07 } \\
\text { to } 2015-15(\%)\end{array}$ & 6.06 & 3.28 & 2.44 & 8.19 & 5.49 \\
\hline
\end{tabular}


It has been observed from Table 6 that the power generated using coal has been increased by 1.29 percentage whereas power generated by using natural gas has been decreased by 0.84 percentage. So, the power stations having increased electric power generation are given additional responsibility of monitoring different regulations like metering, line, load, tariff and other regulations, rather than giving the responsibilities to the power stations generating less electric power. Thus a coordination between different power stations has been established.

\section{Conclusion}

The research work has collected essential data and information from the National Information centre of India. It also proposed an improvised regulated and enforced ORDER technique. The conceptual modelling of a Smart grid is transformed into an architectural model with layered component services through appropriate web services. These software defined control is achieved through the cloud service providers. The basic public and private cloud services are integrated to achieve a hybrid cloud for achieving inter-operable and inter-changeable energy information and security information exchange. The sensitivity and selectivity measures are also proposed and applied across the smart grid layers. The sources of energy sets are grouped as loops with each type of regulatory conditions imposed by one node on the other. By the incorporation of various legal and regulatory standards and compliances, the performance and efficiency of the distributed energy sources are increased significantly. The public and private institutions are responsible for setting the guidelines and laws for the integration of energy sources towards safety and security of the cyber world through a sensitive smart grid.

\section{References}

[1] U.S Department of energy, "The smart grid: An introduction”,Litos strategic communication,November 2008,pp. 17-20 .

[2] Joe M. Understanding the smart grid:features, benefits and costs. US Department of energy, National energy technology laboratory, June 2008, pp. 16-19.

[3] SMB Smart grid strategic group(SG3), IEC Smart Grid Standardization Roadmap. June 2010, Edition 1.0,pp. 98-102.

[4] Math HJB, Jin Z, Francisc Z. Power quality aspects of smart grid. ICREPQ'10, Granada (Spain), March, 2010, pp.1-2.

[5] Kiyoshi N, Kyle EB, Lubomir FB,Michael BD. Complete automation of future grid for optimal real time distribution of renewables. in IEEE march 2015: 1-2.

[6] Central Electricity Authority of India, “Annual Generation Report”, Jan 2017, pp 07- 08.

[7] SumitJha, "Urja app shows powercut”, Financial Express, September2016,

[8] Government of India, Ministry of Power, Central electricity authority, Power sector, January 017,pp.5.

[9] Ministry of Statistics and program implementation, "Energy Statistics 2017," Government of India 2017,pp. 26. 